\title{
Qu'est-ce qui ne va pas avec l'EMC?
}

$\mathrm{D}$ epuis janvier, nous joignons au $7 A M C$ des annonces payées d'un programme d'EMC (mdBriefCase) appuyé par des «subventions d'éducation sans restriction» de sociétés pharmaceutiques. Après avoir reçu des plaintes de lecteurs, nous avons appris que les trousses d'EMC contreviennent aux lignes directrices établies par l'Association médicale canadienne ${ }^{1}$ et l'Accreditation Council for Continuing Medical Education ${ }^{2}$ des États-Unis. Par exemple, ce sont la société d'EMC et le commanditaire, et non l'organisateur du cours, qui choisissent les sujets, conçoivent le contenu du cours (qui, dans certains cas, vise à promouvoir l'utilisation du médicament du commanditaire), choisissent le formateur et ne divulguent pas les conflits d'intérêts d'ordre financier. À l'avenir, nous n'inclurons plus ces encarts d'EMC dans nos envois du journal imprimé.

Une proportion importante et croissante des activités d'EMC (environ $60 \%$ aux États-Unis ${ }^{3}$ ) est issue de commandites commerciales, fournies principalement par les sociétés pharmaceutiques. La responsabilité de déterminer si des activités d'EMC dictées par des intérêts commerciaux sont légitimes et admissibles à des crédits du Collège canadien des médecins de famille et du Collège royal est déléguée (par ces deux collèges) au directeur de l'EMC des facultés de médecine du Canada. Les séries EMC en cause a été approuvées par l'Université de Calgary et a reçu ensuite l'aval de l'Association médicale canadienne, qui l'a hébergée sur son site web.

L'AMC et l'organisme d'agrément des États-Unis permettent à des sociétés commerciales d'appuyer financièrement des activités d'EMC à condition que «les organisateurs soient chargés d'en assurer (...) la validité scientifique,

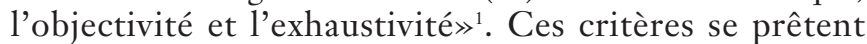
toutefois à interprétation et il s'ensuit que les commanditaires commerciaux interviennent de près. Les subventions d'éducation sans restriction sont faciles à restreindre, à orienter et à manipuler.

Les torts que causent le fait de permettre aux sociétés pharmaceutiques d'orienter les activités d'EMC dépassent-ils toutefois l'avantage de pouvoir les offrir aux médecins à un coût très subventionné, ou gratuitement? Les participants apprendront l'existence de nouvelles lignes directrices et de nouveaux produits - au moins ceux que choisissent de présenter les commanditaires. Le tort que fait le déguisement en contenu adéquat d'un contenu inadéquat et les dommages causés à la réputation de la profession et de ses organismes de régie qui découlent d'une surveillance lacunaire et ambivalente relèguent toutefois cet avantage au second plan. Jouer deuxième violon dans l'orchestre des grandes sociétés pharmaceutiques, ce n'est pas du leadership.
Même si elles coûtent cher, les activités d'EMC ne sont pas un luxe. Les progrès rapides de la médecine et de ses technologies en font une nécessité. Les dépenses des organisateurs et des moniteurs, les documents à distribuer et les aides didactiques, la publicité, les locaux, les déplacements et les séjours, ainsi que les coûts d'opportunité qu'entraîne l'absence du travail, ne sont malheureusement pas insignifiants. Il est tentant de laisser les sociétés pharmaceutiques payer la facture. Tentant, mais compromettant: aussi altruistes puissent-ils être, les commanditaires commerciaux veulent, en bout de ligne, rehausser le profil de leurs produits. Comme il est plus difficile d'avoir un impact sur les ventes à mesure que l'influence des commanditaires est contrôlée plus rigoureusement, le financement que les entreprises consacrent aux activités d'EMC diminuera et finira par disparaître.

La médecine a accepté et accueilli avec plaisir l'obligation de s'autoréglementer et de garantir au public la compétence continue des médecins. Nous n'avons toutefois pas trouvé de moyens de payer. Comme le régime de salariat se répand de plus en plus (souvent conjugué à des plafonds des honoraires), la plupart des médecins ont peu ou pas du tout de moyens financiers de payer leurs activités d'EMC en haussant leurs honoraires ou en travaillant davantage. S'il faut détourner les coûts de l'EMC des commanditaires commerciaux, l'argent devra provenir de la poche des médecins.

Voilà qui est inacceptable dans un régime de médecine socialisée. Au Canada comme ailleurs, nous payons notre système de santé à même les revenus fiscaux généraux, tout comme nous payons la formation des médecins (mais peutêtre pas autant que nous devrions le faire) et leur formation postdoctorale. Il faut reconnaître que l'EMC fait aussi partie de la formation des médecins et trouver un moyen de payer à même les fonds publics, que ce soit par des subventions aux facultés de médecine ou directement aux médecins. Si nous laissons le financement de l'EMC entre les mains du marché, nous constaterons que celui-ci offrira de l'information continue sur les produits et non de l'éducation médicale continue. $-7 A M C$

\section{Références}

1. Association médicale canadienne. Les médecins et l'industrie pharmaceutique (mise à jour 2001) [sommaire de politique]. Ottawa: L'Association;1998. Disponible à : www.cma.ca/staticContent/HTML/N0/11/notre_position /les_medecins_et_l'industrie_pharmaceutique.pdf (consulté le 23 février 2004).

2. Accreditation Council for Continuing Medical Education. Standards for commercial support of continuing medical education, 1992. Disponible à : www .sacme.org/Outside_SACME/documents/standards_commercial_suport.pdf (consulté le 20 février 2004).

3. Relman AS. Defending professional independence. ACCME's proposed new guidelines for commercial support of CME. FAMA 2003;289(18):2418-20. 